\title{
Gastrointestinal Stromal Tumors of The First Jejunal Small Bowel: A Case Report
}

\author{
S Zatir*, A Bouazzaoui, S Bouchakour, N Ougerti, S Laouisset, K Abdellaoui and T Larkam \\ Department of General Surgery, Regional Military Hospital of Oran, Algeria
}

*Corresponding author: Zatir Soufiane, Department of General Surgery, Regional Military Hospital of Oran, Algeria.

To Cite This Article: S Zatir*, A Bouazzaoui, S Bouchakour, N Ougerti, S Laouisset, et al., Gastrointestinal Stromal Tumors of The First Jejunal Small Bowel: A Case Report. 2020 - 11(1). AJBSR.MS.ID.001592. DOI: 10.34297/AJBSR.2020.11.001592.

Received: 㠿 November 23, 2020; Published: 眥 December 01, 2020

\begin{abstract}
EGISTs (gastrointestinal stromal tumors) are rare mesenchymal tumors representing $1 \%$ of primary gastrointestinal cancers with an incidence of 10-20 per million inhabitants [1,2]. They are most common in the stomach (60\%), jejunum / ileum (20-30\%), and rectum. Jejunal involvement is much rarer (3-5\%) [3,4]. The case we report underlines the importance of the decisive role that surgery represents in management and diagnosis.
\end{abstract}

\section{Introduction}

Gastrointestinal stromal tumors (GISTs) are rare mesenchymal tumors that are usually located in the stomach or small intestine. Less than $5 \%$ of GISTs are located outside the gastrointestinal tract: these are extra gastrointestinal stromal tumors (EGIST) $[3,4]$. The widely accepted treatment for GISTs and EGISTs is complete resection (R0) when the tumors are not invading or metastasizing. Their treatment is surgical [5]. Currently, if the entity of stromal tumors is well defined, there remains uncertainties as to their classification according to their degree of malignancy involving long-term follow-up of all patients. The aim of this article is to review the therapeutic approach of digestive stromal tumors and to clarify the management after surgical treatment of these patients.

Case

A 50-year-old patient, with no medical history, consults for a weight loss of $6 \mathrm{~kg}$ and intermittent epigastric discomfort with vomiting. On clinical examination, his doctor found an epigastric mass and anemia. Blood biology shows a mild inflammatory syndrome (CRP at $1.38 \mathrm{mg} / \mathrm{dL}$ (normal: 0.0-0.5 mg / dL)). Two tumor markers were assayed: the CEA is increased to $19 \mathrm{ng} / \mathrm{mL}$ (normal: 0.0-8.0ng / mL), and the CA 19.9 is within the standards. Objective eso-gastro-duodenoscopy (Figure 1) at Treitz's angle of edematous and compressed mucosa of the jejunal mucosa (Figure 1).

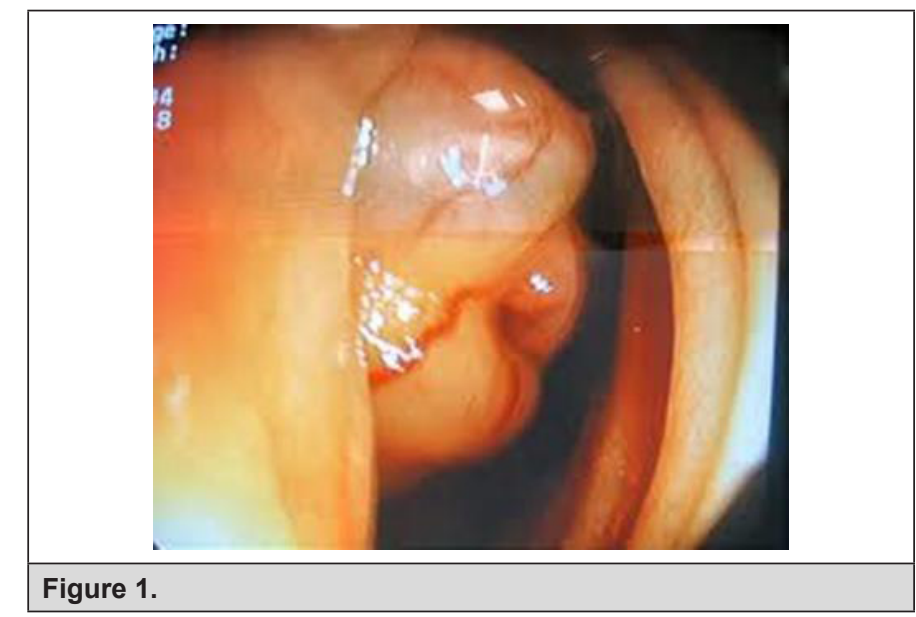

An abdominal CT scan is performed which targets a mass of 9 cm long axis at the expense of the first and second jejunal loop which takes the contrast which remains resectable from the mesenteric vessels (Figure 2). After multidisciplinary consultation, we decided to operate the patient after a preoperative assessment. the patient is admitted to the operating room under general anesthesia, median incision with horse on the umbilicus; the exploration does not find hepatic metastases so the exploration found a mass at the dependence of the 1st and 2 nd jejunal loop, one proceeds to a monobloc resection carrying the 2 nd handle and a terminal anastomosis is performed, closure without drainage (Figure $3 \& 4$ ). 


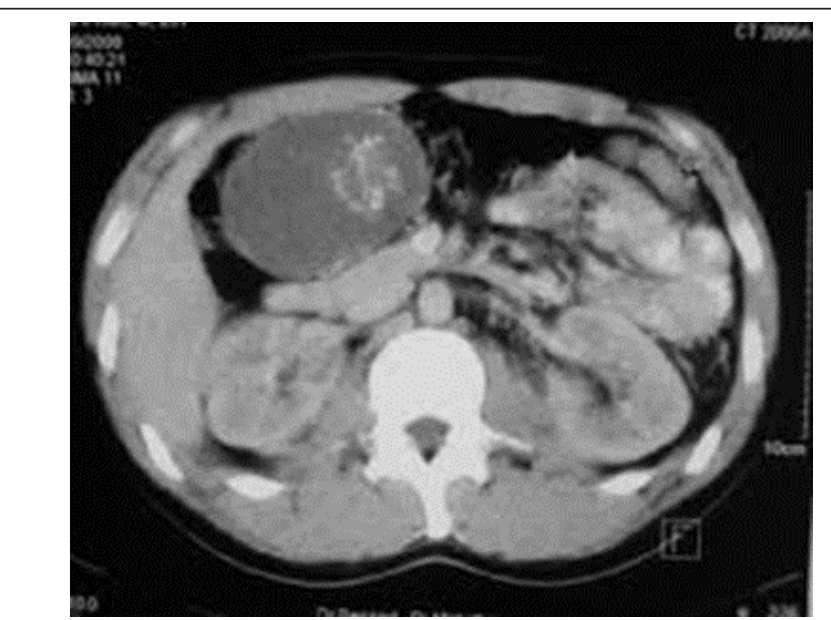

Figure 2.
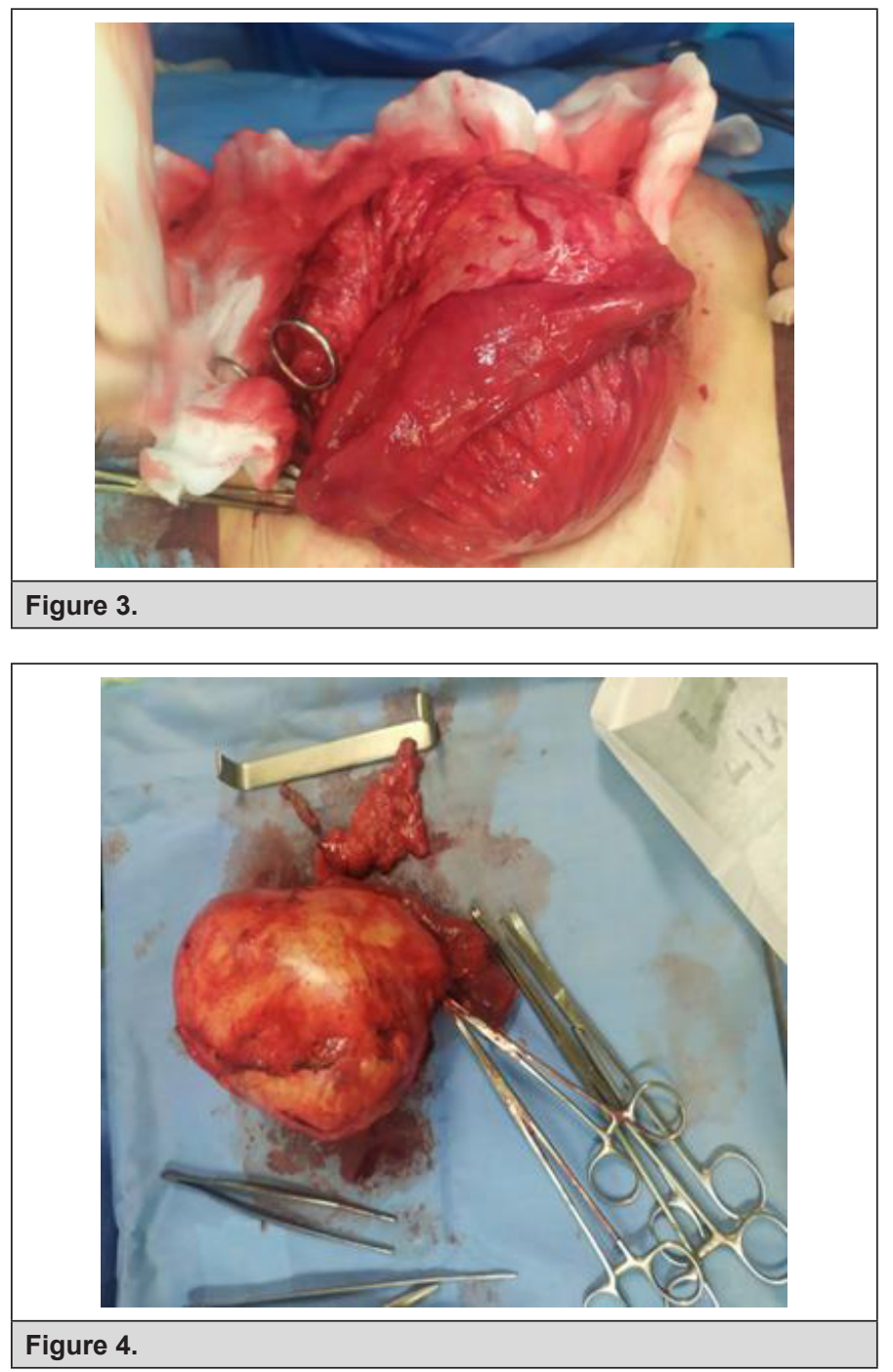

The postoperative consequences were simple, with a resumption of transit on $\mathrm{D}+3$. The patient was declared out on $\mathrm{D}+5$. The histological results allowed the final diagnosis of EGIST derived from the mesentery. Post-operative treatment with imatinib was started (Figure 5).

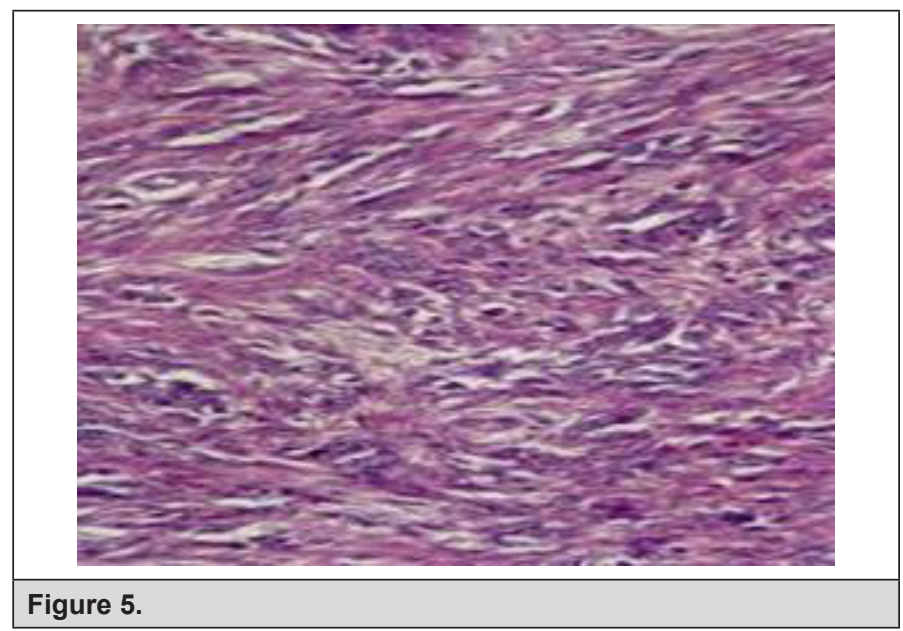

\section{Discussion}

Gastrointestinal stromal tumors (GIST) account for $85 \%$ of mesenchymal tumors of the digestive tract. These rare tumors are most often located in the stomach and small intestine [6,7]. Less than $5 \%$ of GISTs are located outside the gastrointestinal tract, at the level of the mesentery, omentum and / or peritoneum (these are extragastrointestinal stromal tumors: EGIST). Complete singlepiece surgical resection of the tumor (R0 resection) is the only potentially curative treatment for GIST and EGIST [8-9]. In the event of a locally advanced tumor, a large, sometimes mutilating excision is only lawful if the excision is complete, then the alternative of a neo-adjuvant treatment will be retained.

\section{Conclusion}

While complete resection is the widely accepted treatment for EGIST when tumors show no invasion or metastasis to other organs [10-12], the therapeutic strategy for invasive and / or metastatic EGIST has not yet been established. established. To date, [13-16] chemotherapy with imatinib and sunitinib (selective tyrosine kinase inhibitors) is generally used to treat locally advanced and / or metastatic EGIST.

\section{Reference}

1. M Miettinen, L Sobin, J Lasota (2020) Gastrointestinal stromal tumors of the stomach: aclinicopathologic, immunohistochemical, and molecular genetic study of 1765 cases with long-term follow-up. Am J Surg Pathol 29(1): 52-68.

2. JR Goldblum (2002) Gastrointestinal stromal tumors: a review of characteristics morphologic, immunohistochemical, and molecular genetic features. Am J Clin Pathol 117: 49-61.

3. K Kramer, M Siech, J Sträter, AJ Aschoff, D Henne Bruns (2005) GI hemorrhage with fulminant shock induced by jejunal gastrointestinal stromal tumor (GIST) coincident with duodenal neuroendocrine carcinoma (NET) + neurofibromatosis (NF)-Case report and review of the literature. Z Gastroenterol 43(3): 281-288.

4. B Nilsson, K Sjölund, L G Kindblom, J M Meis Kindblom, P Bümming, et al. (2007) Adjuvant imatinib treatment improves recurrence-free survival in patients with high-risk gastrointestinal stromal tumours (GIST). Controlled Clinical Trial 96(11): 1656-1658. 
5. M Miettinen, J Lasota (2001) Gastrointestinal stromal tumors definition, clinical, histological, immunohistochemical, and molecular genetic features and differential diagnosis. Virchows Arch 438(1): 1-12.

6. S Suster (1996) Gastrointestinal stromal tumors: radiologic features with pathologic correlation. Seminars in Diagnostic Pathology 13: 297 313.

7. R C Spiller, Parkins (1983) Recurrent gastrointestinal bleeding of obscure origin: report of 17 cases and a guide to logical management. $\mathrm{Br}$ J Surg 70(8): 489-493.

8. CG Perry, WF Young, SR McWhinney, Thalia Bei, Sotirios Stergiopoulos, et al. (2006) Functioning paraganglioma and gastrointestinal stromal tumor of the jejunum in three women: syndrome or coincidence. Am J Surg Pathol 30(1): 42-49.

9. AD Levy, AM Quiles, M Miettinen, LH Sobin (2005) Gastrointestinal schwannomas: CT features with clinicopathologic correlation. AJR Am J Roentgenol 184(3): 797-802.

10. M SarlomoRikala, M Miettinen (1995) Gastric schwannomas-a clinicopathologic al analysis of six case. Histopathology 27(4): 355-360.

11. MS Kwon, SS Lee, GH Ahn (2002) Schwannomas of the gastrointestinal tract: clinicopathological features of 12 cases including a case of oesophageal tumor compared with those of gastrointestinal stromal tumors and leiomyomas of the gastrointestinal tract. Pathol Res Pract 198(9): 605-613.

12. T Armbrust, M Sobotta, G Ramadori (2007) Follow up of three cases after adjuvant treatment of high risk gastrointestinal stromal tumors with Imatinib. Ann Oncol 18(6): 1123-1125.

13. D Hanahan, RA Weinberg (2000) The hallmarks of cancer. Cell vol 100(1): 57-70.

14. JC Trent II, DJ McConkey, SM Loughlin, MT Harbison, A Fernandez, et al. (1996) Ras signaling in tumor necrosis factor-induced apoptosis. EMBO J 15(17): 4497-4505.

15. T Kubota (2006) Gastrointestinal stromal tumor (GIST) and imatinib. International Journal of Clinical Oncology 11(3): 184-189.

16. R DeMatteo, K Owzar, R Maki et al. (2007) Adjuvant imatinib mesylate increases recurrence free survival (RFS) in patients with completely resected localized primary gastrointestinal stromal tumor (GIST): North American intergroup phase III trial ACOSOG Z9001. in Proceedings of the ASCO Annual Meeting, Chicago, Ill, USA, 10079. 